\title{
Visual regulation of manual aiming: A comparison of methods
}

\author{
Digby ElliotT \\ Liverpool John Moores University, Liverpool, England \\ and McMaster University, Hamilton, Ontario, Canada \\ AND \\ Steve Hansen \\ Brock University, St. Catharines, Ontario, Canada
}

\begin{abstract}
Visual regulation of upper limb movements occurs throughout the trajectory and is not confined to discrete control in the target area. Early control is based on the dynamic relationship between the limb, the target, and the environment. Despite robust outcome differences between protocols involving visual manipulations, it remains difficult to identify the kinematic events that characterize these differences. In this study, participants performed manual aiming movements with and without vision. We compared several traditional approaches to movement analysis with two new methods of quantifying online limb regulation. As expected, participants undershot the target and their movement endpoints were more variable when vision was not available. Although traditional measures such as reaction time, time after peak velocity, and the presence of discontinuities in acceleration were sensitive to the visual manipulation, measures quantifying the trial-to-trial spatial variability throughout the trajectory were the most effective in isolating the time course of online regulation.
\end{abstract}

Since the seminal research of R. S. Woodworth (1899), there has been little doubt that visual-response-produced feedback is important for the online regulation of goaldirected reaching or aiming (see Elliott, Helsen, \& Chua, 2001 , for a review). Specifically, the accuracy and trialto-trial spatial consistency of rapid movement suffers if visual information is eliminated upon movement initiation (e.g., Elliott \& Allard, 1985; Zelaznik, Hawkins, \& Kisselburgh, 1983). Also, visual perturbations during movement execution, such as displacing the visual field (e.g., Hansen, Elliott, \& Tremblay, 2007), modifying the target size (e.g., Hansen \& Elliott, 2009), and changing the characteristics of the visual environment (e.g., Grierson \& Elliott, 2009), all have reliable effects on limb regulation if the performer has enough time to process the new visual information. Of greater controversy than the overall importance of vision are the specific neural and information-processing events that provide the foundation for visual performance advantages. Intimately related to this theoretical issue is the manner in which the processing events responsible for these visual advantages can best be identified and measured.

In a recent review article, Khan et al. (2006) examined some of the ways that movement scientists have attempted to quantify human goal-directed behavior using 1-, 2-, and 3-D data acquisition and analysis protocols designed to capture the characteristics of limb-aiming trajectories.
The most common research strategy has been for investigators to manipulate the availability and/or the type of visual feedback available to a performer and to compare not only performance differences (e.g., speed and accuracy), but also the kinematic characteristics of the limb movements that provide the foundation for these differences. Typically, the measurement protocol is driven by the specific model of limb control under investigation. For example, a study conducted within the framework of Meyer, Abrams, Kornblum, Wright, and Smith's (1988) optimized submovement model would generally focus on the characteristics of individual limb trajectories, with the specific goal of identifying discontinuities in acceleration that might reflect a correction to an initial preplanned movement impulse (e.g., Elliott, Hansen, Mendoza, \& Tremblay, 2004). Investigations designed to identify less discrete regulatory processes are more likely to examine the trial-to-trial spatial variability over multiple aiming trials (Khan, Elliott, Coull, Chua, \& Lyons, 2002). Variability is examined throughout the movement trajectory, and then an attempt is made to determine where, in the course of the trajectory, experimental conditions differ (Hansen, Elliott, \& Khan, 2008).

Rather than adopt a particular theoretical framework that posits late discrete (e.g., Meyer et al., 1988) or early continuous (e.g., Elliott, Carson, Goodman, \& Chua, 1991) control, in this methodological study we selected two ex- 
perimental conditions that have repeatedly yielded large performance differences in terms of endpoint accuracy/ consistency. In other words, we examined manual aiming movements under no-vision and vision conditions. We then compared methodologies designed to identify the source of these performance differences in the movement trajectories. Thus, participants produced a number of goal-directed aiming movements to a small target under conditions in which they had full visual information and under conditions in which liquid crystal goggles eliminated visual information about the limb and movement environment upon movement initiation. We then compared the two experimental conditions using a number of kinematic methods. What is unique about this article is that the same data were used for each of the analytic approaches (cf. Khan et al., 2006). Our goal was to determine not only which methods are most sensitive to visual regulatory processes, but also to identify important commonalities and differences between these standard kinematic approaches to data treatment and analysis. Although we chose to manipulate vision by eliminating it, these same analytic techniques have been and can be used in a number of different contexts to distinguish between preplanned and online limb regulation (see Khan et al., 2006).

With respect to the structure of this article, we begin by describing our data acquisition procedures and general method of data processing and reduction. We then present movement outcome findings that quantify how the two conditions differed in terms of movement speed (i.e., reaction time and movement time) and accuracy/consistency.

Once we have established that our manipulation was effective in producing the expected outcome effects, we examine some of the traditional measures that have been used to isolate the specific processes associated with these effects. These include the symmetry of the limb trajectory, discontinuities in the trajectory, and the effectiveness of these discrete corrective events. Before reporting on each individual measure, we put the use of that specific variable into a theoretical context designed to set the stage for the subsequent discussion of each result.

Next, we present variations of two new approaches to the study of online limb regulation. One approach involves examining how spatial variability in limb position unfolds over the course of the limb trajectory. The other approach involves using correctional methods to determine how well early kinematic events predict later spatial outcomes. Once again, we present the theoretical rationale behind each of these approaches in the context of our findings.

Finally, we make some across-technique comparisons and provide some recommendations designed to guide future work on the visual regulation of goal-directed aiming. We also highlight how the analytic approaches used in the present context might apply more broadly to motor control research.

\section{METHOD}

\section{Participants}

Participants were 9 male and 8 female students and young staff members from McMaster University (mean age $=21.5$ years). All participants were self-declared right-handers and had normal or corrected-to-normal vision.

\section{Apparatus and Procedure}

The target-aiming apparatus was situated on an ordinary tabletop in front of the seated participant. Participants were required to perform a series of discrete aiming movements with a stylus held in their right hands (stylus length $=115 \mathrm{~mm}$ ). They moved from a home position directly in front of them to a 5-mm-square target $40 \mathrm{~cm}$ away. Both the home position and the target were at the midline, so the aiming movement primarily involved elbow extension. An infrared light-emitting diode was placed on the tip of the stylus so that we could track its position using an optoelectric system sampling at $500 \mathrm{~Hz}$ (Optotrak 3020). We chose a 40-cm movement to a small target because previous work from our lab, and elsewhere, has indicated that these specific task requirements would challenge, but not exceed, the limits of visual information processing. Specifically, our goal was to create a situation in which movement times of approximately $400 \mathrm{msec}$ were required for relatively error-free aiming under full-vision conditions.

Participants were instructed to execute their movements as quickly and accurately as possible. A trial began with a verbal "Ready" signal from the experimenter, who simultaneously began the trial by depressing a computer key. The keypress marked the beginning of a 200 - to $1,000-\mathrm{msec}$ variable foreperiod, after which a $100-\mathrm{msec}$ tone sounded, which signaled the participant to begin his/her aiming movement. The optoelectronic recording began at the initiation of the tone. Participants wore a pair of liquid crystal goggles (Milgram, 1987) that were able to change from a clear state to a closed/ translucent state in $3 \mathrm{msec}$. The home position was equipped with a microswitch so that vision could be eliminated at movement initiation by the goggles' changing state (i.e., open to closed) for the no-vision trials. On these trials, the goggles remained closed until the movement was complete, after which they opened to provide the participants with terminal feedback about their performance.

After several practice trials in order to become familiar with the two vision conditions and the overall task requirements, each participant performed 40 experimental trials. These trials were organized into two blocks of 10 trials with vision and two blocks of 10 trials without vision. Vision and no-vision blocks were alternated, with starting order counterbalanced across participants. Prior to each new block of trials, participants were informed as to whether or not vision would be eliminated. Participants were given a short rest between trials, as required.

\section{Initial Data Reduction and Analysis}

We filtered 3-D position data with a Butterworth filter with a low-pass cutoff of $8 \mathrm{~Hz}$. The distance-time profile, as well as the distance profiles in each of the three individual axes, were then differentiated to obtain velocity and differentiated again to obtain acceleration. The velocity profile in the primary direction of movement was used to identify the beginning and the end of each movement and, thus, movement time. Movement initiation was defined as the sample at which instantaneous velocity exceeded $10 \mathrm{~mm} / \mathrm{sec}$ and remained above that criterion for 10 consecutive samples (i.e., $20 \mathrm{msec}$ ). Movement end was defined as the sample at which velocity fell below $100 \mathrm{~mm} / \mathrm{sec}$ and remained there for $20 \mathrm{msec}$ (Hansen, Elliott, \& Khan, 2007). Reaction time was defined as the time between tone onset and movement initiation, whereas movement time was the interval between movement initiation and the termination of the movement.

The spatial position of the stylus at the end of the movement was used to calculate a signed error score in both the primary direction of the movement (i.e., amplitude error) and the direction perpendicular to the prime direction (i.e., directional error) for each trial. This calculation used the center of the target as the reference point. Positive amplitude errors reflect target overshoots, whereas negative errors reflect undershoots. For directional error, negative scores reflect errors to the left and positive scores errors to the right. Means and 
standard deviations of these signed error scores were calculated for each of the two visual conditions and each of the two blocks of trials. These measures of central tendency and trial-to-trial variability are referred to as constant and variable error, respectively.

A custom program was used to identify a number of specific kinematic events, including resultant peak acceleration, peak velocity, and peak deceleration, as well as the 3-D spatial position of the stylus at each one of these specific markers. Along with movement initiation time, it was also possible to calculate the time taken to achieve each one of these kinematic events.

For most single outcome and kinematic measures, separate 2 (vision condition) $\times 2$ (blocks) repeated-measures ANOVAs were conducted on the mean or the standard deviation of the 10 individual trials. For approaches developed to investigate differences between the two visual conditions over the whole course of the movement, an additional factor of kinematic marker or the percentage of the trajectory was included in the analysis. Because some of these analyses involved a complex composite variable such as an $R^{2}$ or a volume based on three different standard deviations, we collapsed over block prior to conducting our initial calculations. This approach was taken to increase the reliability of each datum (e.g., $R^{2}$ values were based on 20 rather than 10 pairs of observations). In these analyses, it is the vision condition $\times$ kinematic marker/percentage of the trajectory interaction that is of most interest, rather than the main effect of vision condition.

Because it was not possible to directly compare dependent variables measured in different units, we also calculated omega-squared values $\left(\omega^{2}\right)$ for each of our significant effects. This statistic provides an indication of the overall variance in the dependent variable explained by the effect, and thus its importance relative to other systematic effects and error (see Tables 1A and 1B). It is worth noting that because the $\omega^{2}$ for any effect represents the proportion of variance that the effect explains within the entire model, $\omega^{2}$ values will be lower when there is a variable in the model that is extremely powerful, such as kinematic marker or percentage of the trajectory.

\section{RESULTS AND DISCUSSION}

\section{Outcome Differences}

Reaction time. The reaction time analysis revealed a main effect of vision condition $[F(1,16)=25.28, p<$ $.001, \omega^{2}=.159$ ], as well as a vision condition $\times$ block interaction $\left[F(1,16)=9.11, p<.01, \omega^{2}=.013\right]$. Overall, participants initiated their movements sooner when they knew that vision would be available during movement execution. This vision/no-vision difference was more pronounced in Block 2 than in Block 1 (Block 1: vision $=282 \mathrm{msec}$, no vision $=314 \mathrm{msec}$; Block 2: vision $=260 \mathrm{msec}$, no vision $=317 \mathrm{msec})$. This finding is similar to previous results from our lab (e.g., Elliott \& Allard, 1985; Hansen, Glazebrook, Anson, Weeks, \& Elliott, 2006; Khan et al., 2002) and has been interpreted to mean that participants take the time to prepare a more precise initial submovement when they know that there will not be an opportunity for online visual regulation during movement execution.

Movement time and error/consistency. The movement time analysis failed to reveal any significant effects. The grand mean was $407 \mathrm{msec}$.

The analyses of amplitude error yielded only main effects of vision condition. The constant error analysis indicated that participants undershot the center of the target when vision was eliminated upon movement initiation $(-4.99 \mathrm{~mm})$, but were accurate when vision was avail-
Table 1A

Omega-Squared Values for the Main Effect of Vision Condition for Variables Examined at a Single Point in Time

\begin{tabular}{ll}
\hline \multicolumn{1}{c}{ Variable } & Omega Squared $\left(\omega^{2}\right)$ \\
\hline Outcome differences & .159 \\
Reaction time & .192 \\
Constant error (amplitude) & .331 \\
Variable error (amplitude) & .301 \\
Variable error (direction) & .098 \\
Proportional time after peak velocity & .105 \\
Discontinuities & .193 \\
Index of error correction effectiveness & .128 \\
$R$ to $z$ score (Elliott et al., 1999) &
\end{tabular}

Table 1B

Omega-Squared Values for the Vision Condition by Kinematic Marker/Percentage Movement Time Interaction for Variables Examined at Multiple Points in the Trajectory

\begin{tabular}{cc}
\hline Variable & Omega Squared $\left(\omega^{2}\right)$ \\
\hline Amplitude variability & .043 \\
Directional variability & .068 \\
3-D variability & .038 \\
\hline
\end{tabular}

able $(-0.09 \mathrm{~mm})\left[F(1,16)=11.91, p<.01, \omega^{2}=.192\right]$. Variable error was also greater under no-vision conditions $(8.04 \mathrm{~mm}$, vision $=4.06 \mathrm{~mm})[F(1,16)=33.28, p<.001$, $\left.\omega^{2}=.331\right]$. These results were not surprising, given the robust nature of this particular visual manipulation (see, e.g., Elliott et al., 2001).

The analysis of directional variable error also revealed a robust main effect of vision condition $[F(1,16)=37.48$, $\left.p<.001, \omega^{2}=.301\right]$. Once again, variability was twice as large under no-vision conditions $(6.29 \mathrm{~mm}$, vision $=$ $3.05 \mathrm{~mm}$ ). For constant error, there was an overall tendency for participants to aim to the right of the target (grand mean $=1.87 \mathrm{~mm}$ ). A significant vision condition $\times$ block interaction $\left[F(1,16)=6.75, p<.05, \omega^{2}=.023\right]$ indicated that this tendency was slightly reduced during Block 2 of no-vision aiming (Block 1: vision $=1.86 \mathrm{~mm}$, no vision $=2.73 \mathrm{~mm} ;$ Block 2 : vision $=2.08 \mathrm{~mm}$, no vision $=$ $0.79 \mathrm{~mm}$ ).

Overall. Overall, participants performed with a greater degree of precision when vision was available for online control during movement execution. This added precision did not cost participants any extra time. In fact, participants took less time to prepare their aiming movements when they knew in advance that vision would be available for online control. In the following section, we examine a number of different approaches used to explain and quantify the nature of vision versus no-vision differences in limb control.

\section{Kinematic Differences}

Symmetry of the trajectory. Many dual-process models of limb control hold that the early portion of a goaldirected aiming movement is under central regulation and that feedback-based regulation becomes more important as the limb approaches the target area (e.g., Woodworth, 1899). A number of investigators have demonstrated that 
when vision is available, performers tend to reach higher peak velocities earlier in the movement than when vision is not available for concurrent limb control (e.g., Elliott, Chua, Pollock, \& Lyons, 1995). This strategy gets the limb into the target area quickly so that more real and proportional time can be spent using visual feedback while the limb is in the target area. Because the time after peak velocity is associated with online control (see Heath, Hodges, Chua, \& Elliott, 1998), a number of investigators have used the symmetry of the velocity profile to index the degree of online regulation. This dual-process approach to limb control predicts that, for the same movement time, participants will spend more time decelerating the limb (i.e., time after peak velocity) than accelerating the limb (i.e., time before peak velocity) when vision is available.

As expected, the analyses of proportional time spent after resultant peak velocity revealed a significant main effect of vision condition $[F(1,16)=26.78, p<.001$, $\left.\omega^{2}=.098\right]($ vision $=.643$, no vision $=.610)$. Presumably, the extra time after peak velocity associated with the fullvision condition reflects the extra processing time required to reduce any discrepancy between the limb and the target position late in the movement (e.g., Carlton, 1992).

Discontinuities in the trajectory and their effectiveness. A number of models of limb control hold that visual regulation is achieved via either single (e.g., Beggs \& Howarth, 1972) or multiple discrete corrections to the movement trajectory (e.g., Keele, 1968; Meyer et al., 1988). Corrections are typically inferred from the presence of discontinuities in the movement trajectory. Although there are minor differences in the way discontinuities are identified (e.g., Chua \& Elliott, 1993, vs. Walker, Philbin, Worden, \& Smelcer, 1997; see also van Donkelaar \& Franks, 1991), generally, researchers have attempted to isolate changes in velocity and acceleration that meet some minimal temporal and amplitude criteria. For example, Chua and Elliott (1993) identified three types of corrections: (1) second zero-crossings in the acceleration profile (second accelerations that are needed when the initial submovement falls short of the target); (2) a negative velocity in the primary direction of the movement (this feature of a velocity profile reflects a reversal that occurs if the initial movement impulse overshoots the target); and (3) a significant deviation in acceleration without a zerocrossing (this type of discontinuity reflects a noticeable change in acceleration sometime after peak velocity). Following Chua and Elliott, these discontinuities had to last a minimum of $70 \mathrm{msec}$ (i.e., temporal criterion) and be at least $10 \%$ of the peak acceleration amplitude (i.e., amplitude criterion; see Chua \& Elliott, 1993, for an explanation for why these specific criteria were chosen).

The notion associated with most discrete models of limb control is that when vision is available, there should be greater probability of one or more corrections within the movement trajectory. Thus, we counted the number of trials in a block with one or more corrections associated with individual aiming movements and compared the two vision conditions. This analysis revealed a modest main effect of vision condition $[F(1,16)=6.74, p<$ $\left..02, \omega^{2}=.105\right]$. Almost half the trials had some sort of discrete correction/discontinuity when vision was available (4.71 trials/block, or $47.1 \%$ ), compared with only one third of the trials when vision was eliminated upon movement initiation (3.35 trials/block, or 33.5\%).

Whether or not there are more corrective submovements associated with full-vision than with no-vision aiming conditions, it is clear that any corrections that do occur should be more error reducing when vision is available. To examine this notion, we employed a procedure developed by Khan, Franks, and Goodman (1998), which they termed the index of error correction effectiveness (IECE). The procedure can be used only when a discrete correction, such as those described earlier, occurs. It involves finding the difference between the position of the limb at the beginning of the corrective process, or early error (EE), and the error at the termination of the movement, or final error (FE). The score is then normalized for the overall degree of error. ${ }^{1}$ Using the notation just presented, the formula is as follows:

$$
\mathrm{IECE}=(\mathrm{EE}-\mathrm{FE}) /(\mathrm{EE}+\mathrm{FE}) .
$$

Following the calculation of a mean IECE for each participant in each condition, we decided to pool data from the two blocks for this analysis because there were 2 participants who had no corrections in one or the other block. This analysis revealed an effect for vision condition $\left[F(1,16)=7.63, p<.02, \omega^{2}=.193\right]$, with corrections associated with the full-vision condition being more error reducing (.870) than corrections made without vision (.814). Taken together with the number of discontinuities analysis, these findings indicate that discontinuities in the trajectory were more than just a by-product of feedforward processes associated with movement planning (e.g., Plamondon \& Alimi, 1997). That is, at least some discontinuities reflect corrective processes associated with the processing of response-associated feedback.

Unfolding spatial variability across multiple repetitions. In recent years, it has become apparent that visual regulation can begin quite early in an aiming movement and that corrective processes are not always discrete in nature (e.g., Elliott et al., 1991; Grierson \& Elliott, 2009; Hansen et al., 2006; Khan et al., 2002; Khan et al., 1998; Proteau \& Masson, 1997), especially when a perturbation of the target changes the physical requirements of the task (e.g., Hansen \& Elliott, 2009; Heath et al., 1998). This type of finding has motivated researchers to consider methodological approaches that do not depend on the identification of discrete discontinuities in aiming profiles. Khan and colleagues have championed an approach to movement analysis that examines trial-to-trial spatial variability as movements unfold (e.g., Khan et al., 2002; Khan et al., 2006; Khan et al., 2003). The primary assumption of this approach is that there is neural-motor noise associated with the execution of any goal-directed movement. This noise will lead to variability in the path of the movement trajectory across multiple attempts to achieve exactly the same movement goal. If these movements unfold in an unregulated fashion, then the degree of spatial variability, 
as indexed by the standard deviation in spatial position, should systematically increase as movement progresses (e.g., the standard deviation of amplitude will increase in proportion to the mean amplitude covered; see Khan et al., 2002). Thus, spatial variability should increase from peak acceleration (PA) to peak velocity (PV) to peak deceleration (PD) and, in fact, be greatest at the termination of the movement (End). However, this outcome would not occur if the unfolding movement is regulated via visual or other types of feedback. In this case, variability should level off, or even decrease, as corrective processes begin to have an impact.

The general approach taken by Khan and colleagues (Khan et al., 2002; Khan et al., 1998; Khan et al., 2003) has been to examine the standard deviation of the limb's spatial position at the specific kinematic markers mentioned above (i.e., PA, PV, and PD) as well as at the end of the movement. When the movements are made in 3-D space (e.g., Khan et al., 2002), the general procedure has been to use resultant kinematic markers as reference points, but to separately examine spatial variability in the primary direction of the movement (i.e., amplitude variability) and perpendicular to the primary direction of the movement (i.e., directional variability). This approach was taken because these two movement dimensions may be controlled separately (see, e.g., Bard, Hay, \& Fleury, 1985; Elliott et al., 2001; Ghez, Gordon, Ghilardi, \& Sainburg, 1995). Thus, our analysis consisted of a 2 (vision condition) $\times$ 4 (kinematic marker: PA, PV, PD, End) repeated measures ANOVA on the standard deviations associated with movement amplitude and those associated with left-right directional position. Of interest was where in the movement trajectory trial-to-trial spatial variability begins to level off or even decrease in the two feedback conditions.

The amplitude variability analysis revealed a main effect of kinematic marker $[F(3,48)=35.90, p<.0001$, $\left.\omega^{2}=.492\right]$ as well as a kinematic marker $\times$ vision condition interaction $\left[F(3,48)=5.19, p<.01, \omega^{2}=.043\right]$. As is evident in Figure 1A, variability increased between PA and PD under both vision and no-vision conditions. However, the increase was greater when vision was available than when it was eliminated upon movement initiation. Participants also exhibited significantly greater variability at PD when vision was available. Variability decreased significantly after PD. However, this reduction in spatial inconsistency was much more dramatic with than without vision (see also Khan et al., 2002). As we also know from the variable error analysis, participants were significantly more consistent at the termination of their aiming movement when they had vision available for online regulation (Tukey's HSD, $p<.05$ ). Presumably, the early differences between vision and no vision reflect visual online control processes designed to amend the errors associated with individual aiming attempts. These feedback-based corrective processes are effective because, by the end of the movement, spatial consistency (i.e., as indexed by variable error) is greater when vision is available. As suggested by Khan et al. (2002), some of the greater inconsistency associated with the full-vision condition at PD may also reflect a less precise movement-planning process. Recall

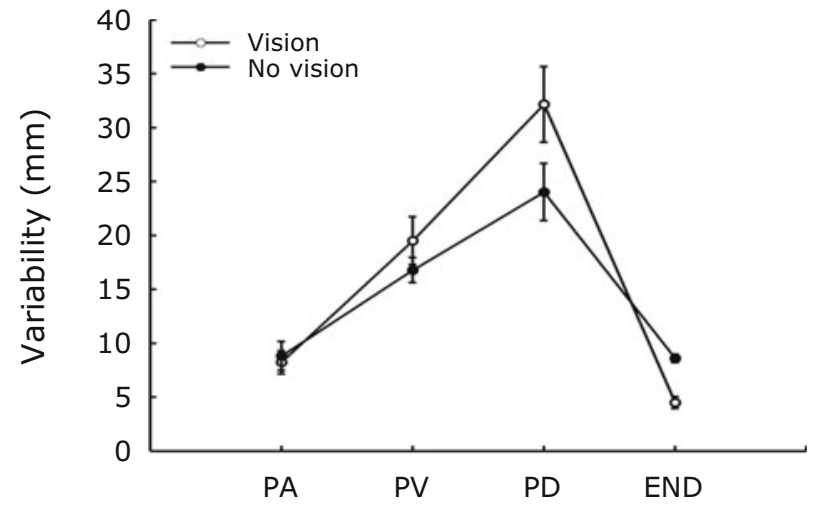

Kinematic Marker

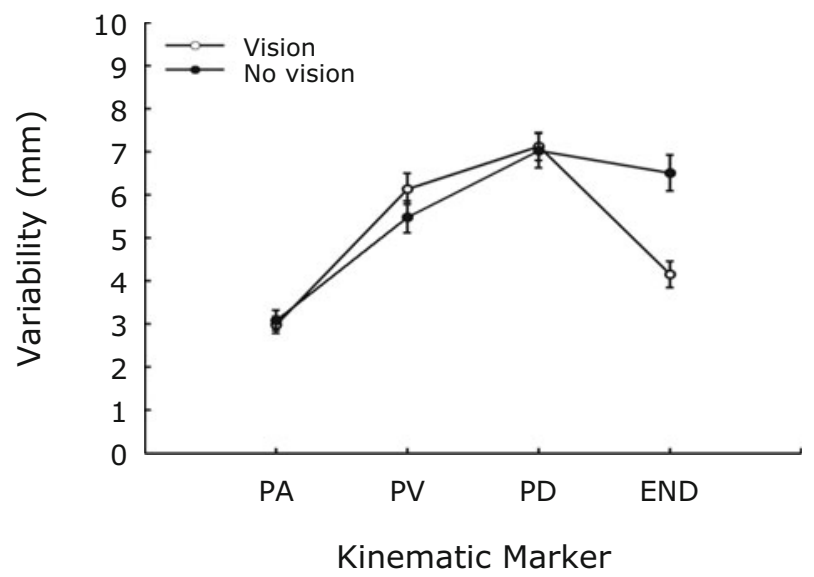

Figure 1. Within-condition spatial variability (in millimeters) of the trajectory of the finger and standard error of the mean at resultant peak kinematic markers (PA, peak acceleration; PV, peak velocity; $P D$, peak deceleration; END, movement endpoint) in the primary (A) and secondary (B) axis of movement for the vision and no-vision conditions.

from the reaction time results that precision planning may not be as necessary when the participant knows that vision will be available for online control (see Hansen et al., 2006).

The directional variability analyses revealed a main effect of kinematic marker $\left[F(3,48)=62.58, p<.001, \omega^{2}=\right.$ .466] and a significant interaction of kinematic marker and vision condition $\left[F(3,48)=13.26, p<.001, \omega^{2}=\right.$ $.068]$. The spatial variability increased between PA and PD under both full-vision and no-vision conditions (Figure 1B). However, variability in the movement direction decreased significantly at the movement endpoint only when vision was available, and only at the termination of the movement was there a significant difference between the two feedback conditions (Tukey's HSD, $p<.05$ ).

This type of spatial variability analysis associated with the work of Khan and colleagues was initially developed to examine 1-D movement and, thus, has limitations when it is used with movement trajectories that vary in three dimensions (see Khan et al., 2006, for a review). This limitation occurs because the $x$-, $y$-, and $z$-axes are defined by 
the researcher conducting the study, and by the associated target-aiming layout. These dimensions may not reflect the control strategy adopted by the performer. Moreover, it is also arbitrary to partition the movement into segments defined by kinematic markers associated with resultant acceleration profiles. For these reasons, Hansen et al. (2008) developed a procedure to examine how 3-D variability unfolds over the entire movement trajectory. Hansen et al.'s (2008) procedure involves normalizing the movement times of an individual performer's aiming attempts and then calculating standard deviations in spatial position for $x, y$, and $z$ at multiple points in the trajectory, to be used as the radii for the ellipsoids. The standard deviations, along with the mean position of the limb, are used to create ellipsoids at specific percentages of the normalized movement times. Essentially, the volume of these ellipsoids is an index, to one standard deviation unit, of the variability in the limb's position in three dimensions, as the movement unfolds. As with the 1-D procedure, the goal is to examine how and where variability increases, and then perhaps decreases, as feedback-based regulation begins to have an impact on the movement trajectory. Our inferential statistics in this case involved treating the ellipsoid volumes as dependent variables and analyzing these volumes using a 2 (vision condition) $\times 25$ (move- ment percentages: $4 \%, 8 \%, 12 \%, \ldots, 100 \%$ ) repeated measures design.

This 3-D variability analysis yielded main effects of vision condition $\left[F(1,16)=5.95, p<.03, \omega^{2}=.012\right]$ and movement percentage $[F(24,384)=30.55, p<.001$, $\left.\omega^{2}=.566\right]$, as well as a vision condition $\times$ movement percentage interaction $\left[F(24,384)=10.01, p<.001, \omega^{2}=\right.$ $.038]$. As is evident in Figure 2, there was very little variability in either movement condition for the first $20 \%$ of the movement (approximately $80 \mathrm{msec}$ ). This point was about the time of PA. After PA, participants were more variable under full-vision conditions. This difference in variability was significant at $32 \%$ of the movement (i.e., approximately $130 \mathrm{msec}$ ), and the two conditions remained different until $64 \%$ of the movement was complete (i.e., approximately $260 \mathrm{msec}$; Tukey's HSD, $p<.05$ ). During limb deceleration, the advantage associated with having full vision became apparent, with participants exhibiting less variability and also less amplitude bias when vision was available (i.e., see variable error and constant error results). Similar to our interpretation of the vision condition $\times$ kinematic marker interaction discussed earlier, the added variability associated with vision, as early as $130 \mathrm{msec}$ into the movement, probably reflects a corrective process associated with the online regulation of the in-

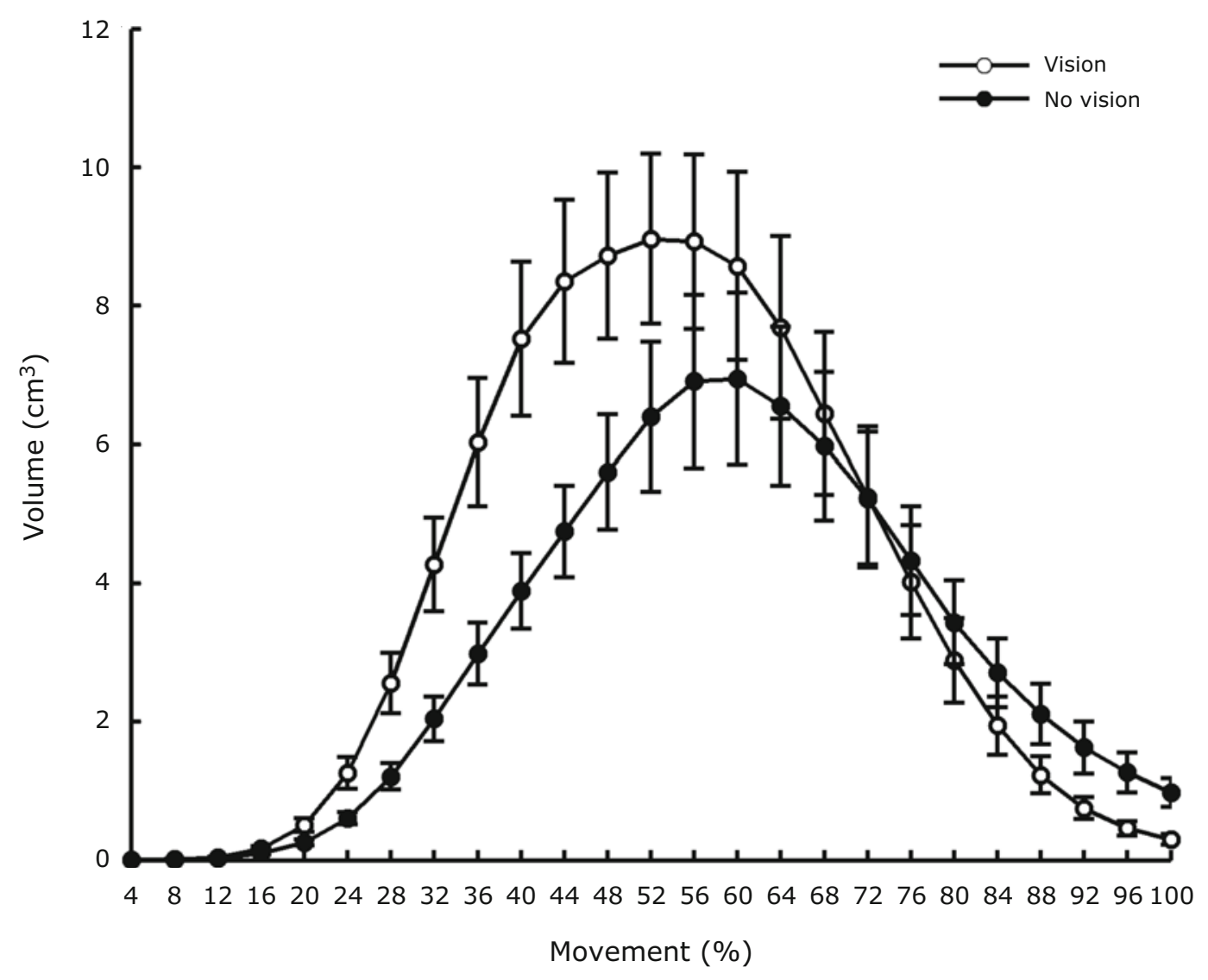

Figure 2. Volume of space (in centimeters ${ }^{3}$ ), the trajectory occupied by the finger, and standard errors of the means as a function of vision condition. 
dividual aiming attempts. The idea is that these processes will reflect the type and magnitude of the aiming error associated with the initial submovement toward the target. The strength of this approach to data analysis is that it not only detects differences between aiming with and without vision, it also precisely identifies where in the movement trajectory these difference begin to be realized.

The relationship between early and late spatial positions. Like some of the earlier approaches that were driven by the two-component explanation of limb control (Woodworth, 1899), Elliott, Binsted, and Heath (1999) reasoned that if a movement is preplanned and then executed without the benefit of online regulation, then early spatial position in a movement trajectory should positively predict late spatial position and thus endpoint error. However, if the limb has traveled farther than intended at PV and the movement error was detected from the sensory feedback, then the movement will be terminated earlier than originally planned. The opposite should be true for visually regulated movements that covered less distance than intended by the acquisition of PV. In an initial study, Elliott et al. (1999) measured the distance covered (i.e., in the primary direction of the movement) by PV and then the distance covered between PV and the end of the movement in the individual movement trajectories. The idea was that if feedback-based regulation is operating, there should be a negative relationship between the distances covered between movement initiation and PV, and PV and the end of the movement. This outcome is exactly what they found. Moreover, this negative relationship was more robust when vision was available than when it was eliminated upon movement initiation (Elliott et al., 1999).

Following the procedure used by Elliott et al. (1999), we calculated these early and late movement amplitudes for each participant in each condition. We then computed a Pearson product-moment correlation coefficient that we then converted to a $z$ score for inferential analysis.

The analysis of these $z$ scores revealed a main effect of vision condition $\left[F(1,16)=16.34, p<.001, \omega^{2}=.128\right]$, with a stronger negative relationship in the vision condition $(z=-1.66)$ than in the no-vision condition $(z=$ $-1.19)$. Both with respect to the probability of a Type I error and the amount of variance explained, this method appears to be at least as powerful in detecting differences between the vision conditions as either the proportion of time after PV or the number of corrections analyses.

Building on the Elliott et al. (1999) procedure, Heath, Westwood, and Binsted (2004) calculated cumulative amplitudes at a number of kinematic markers (i.e., PA, PV, PD) and then separately correlated these amplitudes with the amplitude achieved at the end of the movement. Because the amplitudes were cumulative, early amplitude error was posited to predict late amplitude error if there was limited online regulation of the movement based on feedback (i.e., a robust $r^{2}$ value). However, coefficients of determination ( $r^{2}$ values) should approach zero as the performer is able to reduce any initial aiming bias via online regulation. By examining the degree of relationship between movement amplitudes at three sequential kinematic events, Heath et al.'s (2004) approach has the potential to isolate where in the trajectory feedback-based regulation begins to have an impact. Following Heath et al.'s (2004) procedure, we calculated $r^{2}$ values for each participant for each condition and then used these values as data in a 2 (vision condition) $\times 3$ (kinematic marker: $\mathrm{PA}, \mathrm{PV}, \mathrm{PD})$ repeated measures ANOVA.

This analysis failed to reveal any significant effects. However, the grand mean was low $\left(r^{2}=.127\right)$ and not reliably different from zero. This outcome indicates that for both vision conditions there was a high degree of online control from very early in the movement. Although Heath and colleagues have shown reliable vision-no-vision differences using this method, the main thrust of much of their work has been to identify differences in limb control associated with memory-driven movements. In their protocols, they have also employed multiple target amplitudes (Heath et al., 2004) and conditions in which the target remained illuminated while the limb was occluded (Heath, 2005). In this context, they have found that even very brief no-vision periods prior to movement initiation result in decreased online control. Moreover, in most of their work, they based their $r^{2}$ values on more trials than we have used here. In principle, their method should be as effective in detecting differences between vision and novision conditions as was the Elliott et al. (1999) method upon which it is based. ${ }^{2}$

\section{SUMMARY AND CONCLUSIONS}

Our primary goal was to compare and contrast various methods of data analysis that have been used to examine the visual regulation of goal-directed reaching and aiming movements. Perhaps not surprisingly, all the approaches we used were sensitive enough to detect the difference between vision and no-vision conditions (see Table 1). Some of the approaches, however, entail assumptions that can no longer be met, given the current theoretical state of the literature. For example, it has been clear for more than a decade (see Elliott et al., 1991; Proteau \& Masson, 1997) that, over and above any discrete corrective process associated with limb control (see, e.g., Meyer et al., 1988), there are also more continuous control processes that are not associated with discrete discontinuities in the movement trajectory. The presence and effectiveness of this type of continuous control will simply be missed if a scientist depends on indices of limb control that require the identification of discontinuities in velocity or acceleration. Although these measures are suitable for use when one wants to distinguish discrete from continuous limb control, when used in isolation, they are not sensitive to the latter form of regulation. Moreover, for a methodological point of view, the specific criteria one sets for including a discontinuity can be problematic, and depend not only on the nature of the movement (e.g., movement time, movement amplitude), but also on the noise associated with the particular measurement device. In detecting discontinuities, sampling frequency and filtering procedures are a much bigger issue than when using a more straightforward measure of late limb control, such as the proportion of time after peak velocity (see Hansen, Elliott, 
\& Khan, 2007). In this work, vision condition accounted for very similar amounts of variance in the number of discontinuities and the proportional time after PV analyses (see Table 1). Certainly, the proportion of time after PV is much easier to measure. It may therefore be useful to examine the symmetry of the trajectory first and then proceed to an examination of discontinuities only if one is interested in distinguishing between discrete and continuous online control.

Although the correlational methods discussed at the conclusion of the Results and Discussion section appear to hold some promise, the approaches that involve quantifying changes in trial-to-trial variability over the course of the trajectory appear to be the most useful. These methods were not only effective in detecting vision versus novision differences, they were also sensitive in determining where in the movement trajectory these differences begin to emerge. Thus, they have the potential to provide us with information about the time course of various sorts of feedback processing. The 1-D procedures championed by Khan and colleagues, and variations of these procedures in which movement percentage replaces kinematic marker as an independent variable, are powerful if movement occurs in only one dimension or if the researcher wants to examine amplitude and direction independently. However, for 3-D movements, the Hansen et al. (2008) protocol appears to provide the most detailed information about both early and late limb control. As we have suggested elsewhere (Hansen et al., 2008), 1- and 3-D protocols examining limb control do not need to be mutually exclusive. For example, if the task involves 3-D displacement and the researcher is interested in examining amplitude and direction separately, the 3-D procedure can be used initially to identify overall differences between conditions, and the individual axes can be examined subsequently to determine whether the differences detected in the overall analysis can be explained to a greater extent by regulation in a particular axis.

In this study, we chose to examine online control by manipulating the availability of vision. However, these same procedures, or variations of them, can be used in many other motor control contexts. For example, in other work, we have used the Hansen et al. (2008) spatial variability protocol to examine how movement trajectories change following a perturbation of target size and target position at movement initiation (Hansen \& Elliott, 2009). The same protocol can be used with grasping movements to quantify changes in grip aperture as a reaching movement unfolds (Hansen et al., 2008).

Although an investigator's particular theoretical framework is always going to have some impact on the way 3-D limb trajectory data are reduced and analyzed, we hope that the methodological comparisons that we have made here give some insight into the strengths and weaknesses of various approaches. In our own lab, we are currently relying less and less on identifying discrete discontinuities in limb trajectories and using approaches that quantify changes in not only variability but also central tendency in the limb trajectory, as the movement unfolds. Although we have not discussed central tendency in the context of this article, it becomes a more important determinant of performance when the perturbation associated with the independent variable is directional (e.g., shifting real or perceived target or limb position), as opposed to simply creating variability by eliminating specific sources of information (see Grierson \& Elliott, 2009; Hansen, Elliott, \& Tremblay, 2007; Mendoza, Elliott, Meegan, Lyons, \& Welsh, 2006).

\section{AUTHOR NOTE}

This research was supported by the Natural Sciences and Engineering Research Council of Canada and the Research Institute for Sport and Exercise Sciences at Liverpool John Moores University. Correspondence relating to this article should be directed to D. Elliott, Department of Kinesiology, 1280 Main St. West, McMaster University, Hamilton, ON, L8S 4K1 Canada (e-mail: elliott@mcmaster.ca).

\section{REFERENCES}

BARD, C., HAY, L., \& Fleury, M. (1985). Role of peripheral vision in the directional control of rapid aiming movements. Canadian Journal of Experimental Psychology, 39, 151-161. doi:10.1037/h0080120

Beggs, W. D. A., \& HowarTh, C. I. (1972). The accuracy of aiming at a target: Some further evidence for a theory of intermittent control. Acta Psychologica, 36, 171-177. doi:10.1016/0001-6918(72)90001-7

Carlton, L. G. (1992). Visual processing time and the control of movement. In L. Proteau \& D. Elliott (Eds.), Vision and motor control (pp. 3-31). Amsterdam: North-Holland.

Chua, R., \& Elliott, D. (1993). Visual regulation of manual aiming. Human Movement Science, 12, 365-401. doi:10.1016/0167 -9457(93)90026-L

Elliott, D., \& Allard, F. (1985). The utilization of visual feedback information during rapid pointing movements. Quarterly Journal of Experimental Psychology, 37A, 407-425. doi:10.1080/ 14640748508400942

Elliott, D., Binsted, G., \& Heath, M. (1999). The control of goal-directed limb movements: Correcting errors in the trajectory. Human Movement Science, 18, 121-136. doi:10.1016/S0167 -9457(99)00004-4

Elliott, D., Carson, R. G., Goodman, D., \& Chua, R. (1991). Discrete versus continuous visual control of manual aiming. Human Movement Science, 10, 393-418. doi:10.1016/0167-9457(91)90013-N

Elliott, D., Chua, R., Pollock, B. J., \& Lyons, J. (1995). Optimizing the use of vision in manual aiming: The role of practice. Quarterly Journal of Experimental Psychology, 48A, 72-83. doi:10.1080/14640749508401376

Elliott, D., Hansen, S., Mendoza, J., \& Tremblay, L. (2004). Learning to optimize speed, accuracy, and energy expenditure: A framework for understanding speed-accuracy relations in goal-directed aiming. Journal of Motor Behavior, 36, 339-351. doi:10.3200/ JMBR.36.3.339-351

Elliott, D., Helsen, W. F., \& Chua, R. (2001). A century later: Woodworth's (1899) two-component model of goal directed aiming. Psychological Bulletin, 127, 342-357. doi:10.1037/0033-2909.127.3.342

GheZ, C., Gordon, J., Ghilardi, M. F., \& SaInburg, R. (1995). Contributions of vision and proprioception to accuracy of limb movements. In M. S. Gazzaniga (Ed.), The cognitive neurosciences (pp. 549-564). Cambridge, MA: MIT Press.

Grierson, L. E. M., \& Elliott, D. (2009). Goal-directed aiming and the relative contribution of two online control processes. American Journal of Psychology, 122, 309-324.

HANSEN, S., \& ElliotT, D. (2009). Three-dimensional manual responses to unexpected target perturbations during rapid aiming. Journal of Motor Behavior, 41, 16-29.

Hansen, S., Elliott, D., \& Khan, M. A. (2007). Comparing derived and acquired acceleration profiles: 3 -D optical electronic data analyses. Behavior Research Methods, 39, 748-754.

Hansen, S., Elliott, D., \& Khan, M. A. (2008). Quantifying the variability of three-dimensional aiming movements using ellipsoids. Motor Control, 12, 241-251. 
Hansen, S., Elliott, D., \& Tremblay, L. (2007). On-line control of discrete action following visual perturbation. Perception, 36, 268-287. doi:10.1068/p5629

Hansen, S., Glazebrook, C. M., Anson, J. G., Weeks, D. J., \& ElLIOTT, D. (2006). The influence of advance information about target knowledge and visual feedback on movement planning and execution. Canadian Journal of Experimental Psychology, 60, 200-208. doi:10.1037/cjep2006019

HeAth, M. (2005). Role of limb and target vision in the online control of memory-guided reaches. Motor Control, 9, 281-311.

Heath, M., Hodges, N. J., Chua, R., \& Elliott, D. (1998). On-line control of rapid aiming movements: Unexpected target perturbations and movement kinematics. Canadian Journal of Experimental Psychology, 52, 163-173. doi:10.1037/h0087289

Heath, M., Westwood, D. A., \& Binsted, G. (2004). The control of memory-guided reaching movements in peripersonal space. Motor Control, 8, 76-106.

Keele, S. W. (1968). Movement control in skilled motor performance. Psychological Bulletin, 70, 387-403. doi:10.1037/h0026739

Khan, M. A., Elliott, D., Coull, J., Chua, R., \& Lyons, J. (2002). Optimal control strategies under different feedback schedules: Kinematic evidence. Journal of Motor Behavior, 34, 45-57. doi:10.1080/00222890209601930

Khan, M. A., Franks, I. M., Elliott, D., Lawrence, G. P., Chua, R., Bernier, P.-M., ET AL. (2006). Inferring online and offline processing of visual feedback in target directed movements from kinematic data. Neuroscience \& Biobehavioral Reviews, 30, 1106-1121. doi:10.1016/j.neubiorev.2006.05.002

Khan, M. A., Franks, I. M., \& Goodman, D. (1998). The effect of practice on the control of rapid aiming movements: Evidence for an interdependence between programming and feedback processing. Quarterly Journal of Experimental Psychology, 51A, 425-444. doi:10.1080/713755756

Khan, M. A., Lawrence, G., Fourkas, A., Franks, I. M., Elliott, D., \& Pembroke, S. (2003). Online versus offline processing of visual feedback in the control of movement amplitude. Acta Psychologica, 113, 83-97. doi:10.1016/S0001-6918(02)00156-7

Mendoza, J. E., Elliott, D., Meegan, D. V., Lyons, J. L., \& Welsh, T. N. (2006). The effect of the Müller-Lyer illusion on the planning and control of manual aiming movements. Journal of Experimental Psychology: Human Perception \& Performance, 32, 413-422. doi:10.1037/0096-1523.32.2.413

Meyer, D. E., Abrams, R. A., Kornblum, S., Wright, C. E., \& Smith,
J. E. K. (1988). Optimality in human motor performance: Ideal control of rapid aimed movements. Psychological Review, 95, 340-370. doi:10.1037/0033-295X.95.3.340

MilgRam, P. (1987). A spectacle-mounted liquid-crystal tachistoscope. Behavior Research Methods, Instruments, \& Computers, 19, 449-456.

Plamondon, R., \& Alimi, A. M. (1997). Speed/accuracy trade-offs in target directed movements. Behavioral \& Brain Sciences, 20, 279349.

Proteau, L., \& Masson, G. (1997). Visual perception modifies goaldirected movement control: Supporting evidence from a visual perturbation paradigm. Quarterly Journal of Experimental Psychology, 50A, 726-741. doi:10.1080/027249897391865

van Donkelaar, P., \& Franks, I. M. (1991). The effects of changing movement velocity and complexity on response preparation: Evidence from latency, kinematic and EMG measure. Experimental Brain Research, 83, 618-632. doi:10.1007/BF00229840

Walker, N., Philbin, D., Worden, A., \& Smelcer, J. B. (1997). A program for parsing mouse movements into component submovements. Behavior Research Methods, Instruments, \& Computers, 29, 456-460.

WoODWORTH, R. S. (1899). The accuracy of voluntary movement. Psychological Review, 3(Suppl.), 1-119.

Zelaznik, H. N., Hawkins, B., \& Kisselburgh, L. (1983). Rapid visual feedback processing in single-aiming movements. Journal of Motor Behavior, 15, 217-236

\section{NOTES}

1. Unlike the Khan et al. (1998) index, which was calculated in only one dimension, the error scores here reflect the 3-D radial distance between the marker on the stylus and the center of the target.

2 . In our calculations of the initial $r$ values, many of the values were in fact negative. This would be expected due to random variation if the true state of affairs is the $r=0$. By squaring negative $r$ values, one shifts any distribution with a mean of zero to something positive. This approach also artificially reduces the variability of the distribution. Given that the goal is to statistically detect a difference between an $r$ value that reflects some degree of covariation and a theoretical value of zero (i.e., independence), it would be more powerful to use the $r$ values or their transform ( $z$ scores; see, e.g., Elliott et al., 1999) than $r^{2}$ values.

(Manuscript received February 3, 2010; revision accepted for publication June 2, 2010.) 